\title{
Spatial 3d Direct Visualization of Atoms, Molecules and Chemical Bonds
}

\author{
Olexandr Kucherov ${ }^{1,}$, , Alexandr Rud ${ }^{2}$, Viktor Gubanov ${ }^{3}$, Mykola Biliy $^{3}$ \\ ${ }^{1}$ Institute of Structural Information Technology, Kyiv, Ukraine \\ ${ }^{2}$ G. V. Kurdyumov Institute for Metal Physics of the N. A. S. of Ukraine, Kyiv, Ukraine \\ ${ }^{3}$ Department of Experimental Physics, Faculty of Physics, Taras Shevchenko National University of Kyiv, Kyiv, Ukraine
}

Email address:

O.Kucherov@i.ua(O. Kucherov)

${ }^{*}$ Corresponding author

\section{To cite this article:}

Olexandr Kucherov, Alexandr Rud, Viktor Gubanov, Mykola Biliy. Spatial 3d Direct Visualization of Atoms, Molecules and Chemical Bonds. American Journal of Applied Chemistry. Vol. 8, No. 4, 2020, pp. 94-99. doi: 10.11648/j.ajac.20200804.11

Received: March 19, 2020 Accepted: April 2, 2020; Published: August 13, 2020

\begin{abstract}
The paper presents the further development of direct visualization of individual atoms, molecules with chemical bonds by spatial 3d electron cloud densitometry. Direct visualization of small objects studied by chemistry is made possible by the discovery of the plane wave amplitude deformation in accordance with the electron cloud density. Accordance with this affect, an atom begins to illuminate, depicting its own form! A quantum mechanical theory of the effect is given. As a result, it was possible to trace a chemical reaction with a change in the chemical bonds, geometry molecules, and distances between the atoms. The spatial $3 \mathrm{~d}$ picoscope is used to show the structure of crystalline graphite formed by the $s p^{2}$ chemical bonds of carbon atoms, and a system of weak $\pi$-bonds that connects the layers of graphite. This device confirmed the flat structure of single-layer graphene and allowed to detect a two-layer hexagonal diamond system that was previously un known. This material is proposed to designate gubanite. A scan be seen from the spatial $3 \mathrm{~d}$ snapshot, as the graphene layers converge, the upper graphene $\pi$-electron clouds connect to the lower graphene $\pi$-electron clouds to form a two-layer diamond gubanite, the spatial $3 \mathrm{~d}$ model of which is shown. It should lead to the appearance of visual chemistry.
\end{abstract}

Keywords: Direct Image of Electron Clouds, Electron Cloud Densitometry, Graphene, Two-layered Diamond, Spatial 3d Picoscope, Visualization of Molecules, Visualization of Chemical Bonds, Visual Chemistry

\section{Introduction}

Solid carbon exists in several allotropic forms: graphite, diamond, graphene, nanotubes, fullerenes, vitreous carbon, coke, coal etc. However, if X-ray diffraction gives accurate crystallographic information, spectroscopic methods give quantitative information on carbon content and the ratio of $s p^{2} / s p^{3}$ bonds $[1,2]$. It is shown that in-plane $s p^{2}$ bonds are strained and distorted after ball milling because $s p^{3}$ bonds are introduced [3]. Much of what we know about the microstructures of these materials is obtained by use of picoscope [4]. Picoscope has an unmatched ability to give a direct visualization of the atomic structure together with chemical bonds. There is one fundamental problem in the study of nanostructures and in the management of nanotechnology: so far no one has yet seen an electron that determines shapes of all chemical objects. As is known, the molecules shapes are determined by the electron clouds created by the motion of the electron around the nucleus and determined by laws of quantum mechanics. The problem with the visualization of the electron shells of atoms is that in nature it is unknown rays that can be reflected from an electron cloud and thus transmit its shape. Therefore, instead of obtaining an image of an object, in this case an electron, a fundamentally new method of obtaining an image of the electron cloud density is proposed.

\section{The Principle of Electron Cloud Densitometry}

Molecules have no body, therefore optics, both 
photonic and electronic, do not allow seeing them. This is an indisputable law of nature. But, where the laws of optics do not work, the laws of quantum mechanics apply. The fundamental basis of quantum mechanics is the Heisenberg uncertainty principle, which captures the following fact: an electron beam passed through a measuring device carries information about that device. And here the question arises: could the electron cloud be the "measuring device"? O. P. Kucherov and S. E. Lavrovsky [5] found that the passage of an electron beam through the electron cloud also transmits to the beam information about the structure of the electron clouds of individual atoms, molecules with chemical bonds.

The property of atoms to shift the electron beam is shown in Figure 1(b) compared to the property of a substance to absorb the rays, shown in Figure 1(a).

The difference between these two phenomena can be schematically illustrated as follows. First, let the beam have 11 rays of 3 electrons each, then in all 33 electrons.

The substance, Figure 1(a), consists of a large number of atoms and absorbs electrons. As a result, the beam bears losses. These losses increase with increasing sample thickness. Figure 1(a) shows that only 20 electrons were overcome by the sample. Moreover, on the periphery, where the substance is absent, all 3 electrons in each ray passed freely. As a result, a negative of the test sample appeared on the receiver.

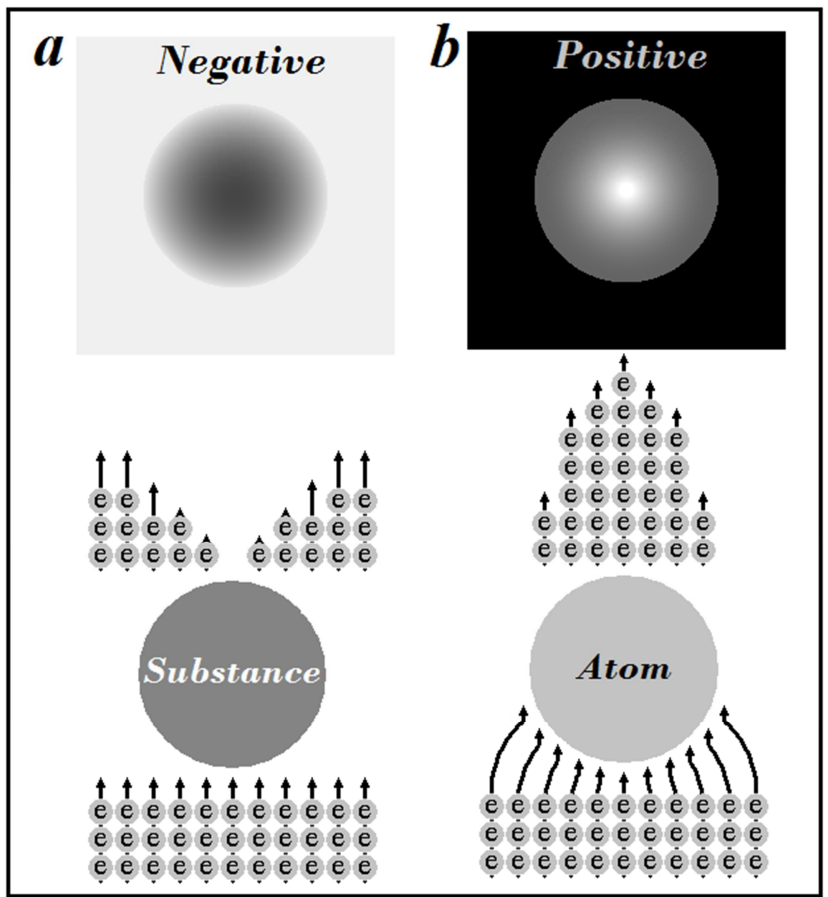

Figure 1. Absorption of the electron beam by a substance gives negative image (a). Deformation of the electron beam by atoms gives positive image (b).

When the object under study has the size of an atom, figure 1(b), electron rays pass through the atom without absorption. However, as a result of the electron beam shifting effect, atoms attract an electron beam without changing their speed (both amount and direction).

On the periphery, where the electron cloud is absent, the rays have completely disappeared, shifting their electrons to the center.

The intensity of the rays increases in the center, where the thickness of the electron cloud is the maximum. The result is the positive image. The atom begins to illuminate, depicting its own form!

\section{The Theory of Electron Cloud Densitometry}

The basis of quantum mechanics is the statement that the wave function $\Psi(q)$, with coordinates $q$, describe the state of the system and the square modulus of this function determines the probability to find a system in the volume $d q$ :

$$
\rho(q)=|\Psi|^{2} \text { dq. }
$$

Consider the proposed quantum microscopy system consistent of two subsystems. The electron clouds of the testing sample are the first subsystem with electrons coordinates $q$. The electron beam with the coordinates $p$ is the second subsystem. A wave function of the complete system is $\Psi(q, p)$.

In this case, the following integral defines the probability $\mathrm{I}(x, y)$ to find the electron at point $x, y$ of the microscope screen:

$$
\mathrm{I}(\mathrm{x}, \mathrm{y})=\iint \Psi(\mathrm{q}, \mathrm{p}) \Psi^{*}(\mathrm{q}, \mathrm{p}) \mathrm{dzdt}
$$

where $t_{0}$ is the start time of the exposure; $t_{l}$ is time completion exposure; $z_{0}$ is the lower plane of the sample; $z_{\max }$ is the upper surface of the sample.

Based on the principle of superposition, the wave function of the system $\Psi(q, p)$ represents the product of the wave function of electrons $\Psi q(q)$ of the sample and radiation $\Psi p(p)$ :

$$
\Psi(\mathrm{q}, \mathrm{p})=\Psi \mathrm{p}(\mathrm{p}) \Psi \mathrm{q}(\mathrm{q})
$$

As is known the wave function of the electron beam is a plane wave:

$$
\Psi(\mathrm{x}, \mathrm{y}, \mathrm{z})=\left(\mathrm{j} / \mathrm{v}_{\mathrm{z}}\right) \mathrm{e}^{\mathrm{jkz}},
$$

where $j$ is the density of electrons in the plane; $k$ is constant for a plane wave; $v_{z}$ is the electrons speed along the axis $z$.

Substituting expression (4) into (2) and take the integral over time, we get the following expression:

$$
\mathrm{I}(\mathrm{x}, \mathrm{y})=\int \rho(\mathrm{x}, \mathrm{y}, \mathrm{z}) \mathrm{dz},
$$

where $\rho(x, y, z)$ is the probability to find an electron in the volume $d q$ of the sample, which satisfies the normalization condition:

$$
1=\iiint \rho(x, y, z) d x, d y, d z .
$$

Note that the normalization condition (6) must be fulfilled for each electron. 
Thus, we found the confluence electron effect $I(x, y)$ as the function of the structure of the electron clouds in the sample $\rho(x, y, z)$ through which the electron beam passes.

Consider this effect. At first sight, expression (5) seems very complicated. Indeed, in order to calculate the probability $\rho(x, y, z)$, it is necessary to take into account all the electrons in the sample under study, and in one gram of substance there are $12 \times 10^{23}$ electrons (charge multiplied by the Avogadro constant). However, with careful study, we can simplify and easily interpret expression (5).

Consequence 1 . The integral intensity of the electron beam at the entrance to the sample $\left(z=z_{0}\right)$ and at the output from it $\left(z=z_{\text {max }}\right)$ is constant.

This statement follows from the generally accepted condition in quantum mechanics for the normalization of the probability $\rho(x, y, z)(6)$ over all atoms inside the sample.

Consequence 2. If there are no electron clouds in its path from $z_{0}$ to $z_{\max }$ at some point $\left(x_{1}, y_{1}\right)$ the intensity $I\left(x_{1}, y_{1}\right)$ of the electron beam is zero.

Indeed, the integral (5) will be zero if at the point $\left(x_{1}, y_{1}\right)$ the function $\rho\left(x_{1}, y_{1}, z\right)=0$ at all points from $z_{0}$ to $z_{\max }$.

It follows from consequence 1 and 2 that the sample shifts the electron beam. The beam shifts from the place where there is no electron cloud $\rho\left(x_{1}, y_{1}, z\right)=0$ to the place where it exists $\rho\left(x_{1}, y_{1}, z\right)>0$. This is the shifting electron effect.

Using expression (5), we will calculate the image $I(x, y)$ of single atom.

The density distribution of electrons in an atom determines by the Thomas - Fermi method (E. Fermi, L. Thomas, 1927), who works for atoms of any complexity. The method uses quasi-classical approximation: each atom of the periodic table there is a definite radius $\mathrm{r}$ (Bohr radius), so at the distances $a<r$ electron density is large and is about the same, and at the distance $a>r$ electron density is low and attenuated with exponential speed. According to the method, the electrons density in the atom takes the following form:

$$
\rho(q)=C(q)+\eta(q),
$$

where $C$ is constant for $a<r$ and is zero for $a>r ; \eta(q)$ is small compared with the $C$. We substitute the electrons density in equation (5).

As a first approximation neglect small amount put $\eta(q)=0$. As a result, the integral (5) with (7) takes the following form:

$$
\mathrm{I}(\mathrm{x}, \mathrm{y})=(\mathrm{jn} / \sigma) \int \mathrm{dz},
$$

where $n$ is the number of electrons in cloud; $\sigma$ is the total electron cloud volume which satisfies the normalization condition (6).

In expression (8), the integral gives the thickness of the electron cloud at the point $x, y$, we denote it by $Z(x, y)$. Thus, for the ray passing through the atom, we finally obtain:

$$
\mathrm{I}(\mathrm{x}, \mathrm{y})=\mathrm{jnZ}(\mathrm{x}, \mathrm{y}) .
$$

Thus, we obtained a law of the electron beam shifting by an atomic electron cloud. The law relates the deformation of the plane wave amplitude $j$ in accordance with the electron cloud density $Z(x, y)$. The law states that the intensity of the electron beam passing through the atom is directly proportional to the thickness (path length) of the electron cloud of the atom at the point of the beam passage.

The law can be applied to visualize the forms of atoms, molecules and chemical bonds; studying their conformation, oscillating and rotational movements; measuring the current of conducting properties.

In this case, we have the electron beam shifting effect. The atom is illuminated by displacement of the electron beam from the edge of the atomic cloud to the center in such a way that the total beam intensity remains constant.

\section{Picoscop}

Picoscope was used to obtain a direct image of the electron shells of atoms, molecules and chemical bonds. The image was obtained on a high-resolution electron microscope (JEOL JEM-2100F) with subsequent conversion to direct electron density imaging using the method [6], Patent of Ukraine No. 115602 .

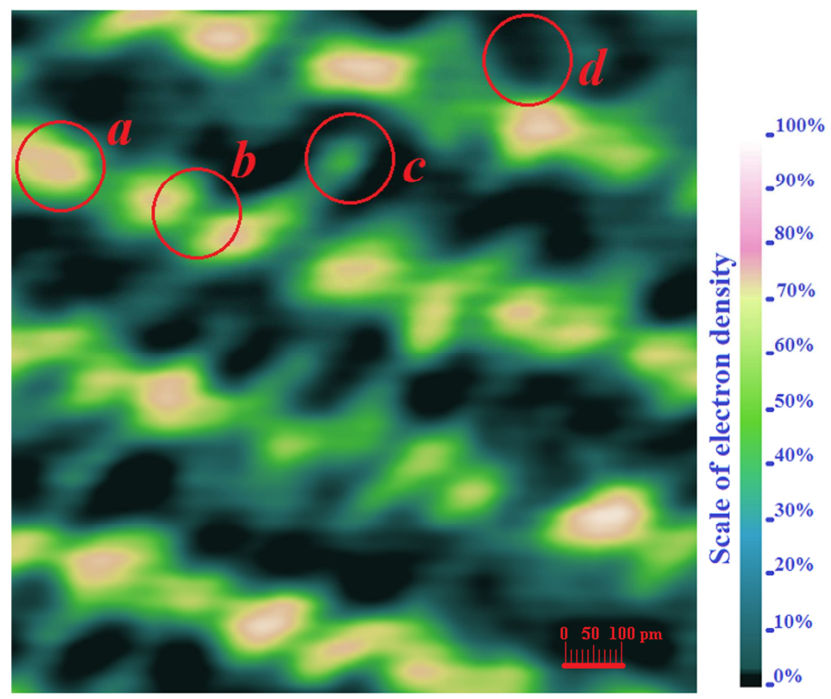

Figure 2. A direct image of graphite. Each carbon atom has its own shape and position (a). All carbon atoms are connect through the chemical $s p^{2}$ bonds (b) and createthe $\pi$-electron clouds (c). The atoms with their chemical bonds are separated by a dark space (d). On the right is the scale of electron cloud density.

Figure 2 shows a direct image of graphite. The scale of electron density is shown on the right in accordance with the formula (9). The distance between the graphite layers is 335 pm (picometrs) and between the atoms is $128 \mathrm{pm}$, which corresponds to the encyclopedia data. The $\pi$ - electron clouds look like elongated blue sleeves which connect with layers of graphite. Due to the new tool, we were able to see tens of thousands of $\pi$ - electron clouds and found them to be specific objects with their own characteristics. In graphite, they look like "sleeves" with a thickness of about $80 \mathrm{pm}$ and a length of about $160 \mathrm{pm}$. The clouds are oriented at an angle of $66^{\circ}$ to the graphite layer. 


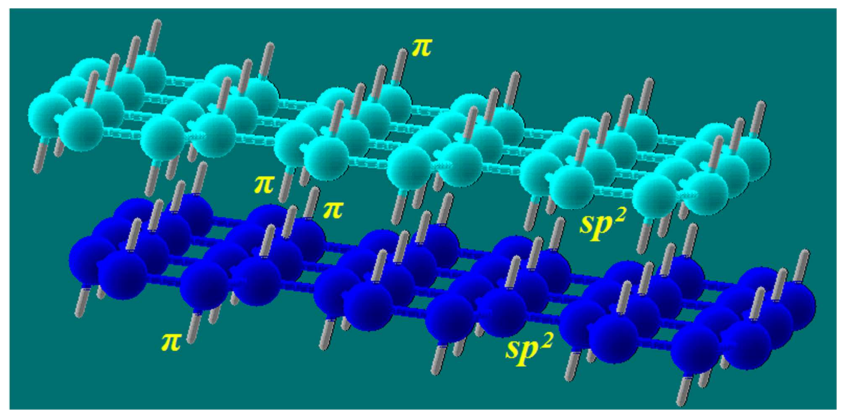

Figure 3. Model of two layers of graphene.

Figure 3 shows a spatial 3d model of two graphene layers adjacent. Each carbon atom is bonded to three adjacent atoms in a way that creates a hexagonal lattice. A fourth valence electron creates the $\pi$ - electron clouds.

\section{Spatial 3d Picoscop}

To derive the formula of spatial $3 \mathrm{~d}$ picoscope, we take a layer of atoms $a$ and $c$ lying in the $x, y$ plane, and one atom $b$ that overtops over the plane at $a$ height $\Delta z$. A distance between the atoms is $d$, as shown in Figure 4.

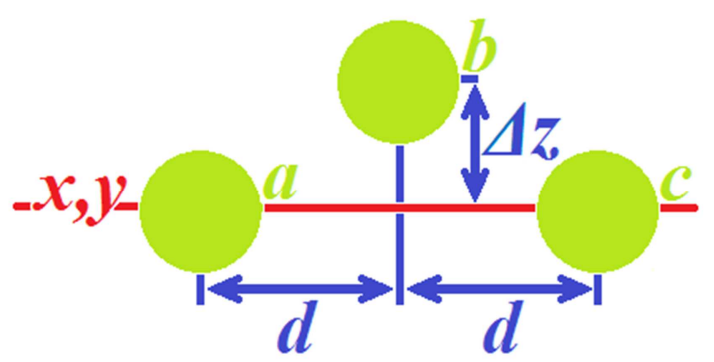

Figure 4. The principle of operation of the spatial 3d picoscope.

Thus, for an electron beam passing through atoms lying at different levels, the formula (9) for the spatial $3 \mathrm{~d}$ picoscope takes the following form:

$$
I(x, y, z)=j n Z(x, y)(1+\Delta z / d) .
$$

The spatial $3 \mathrm{~d}$ picoscope formula allows to measure all three atoms coordinates $x, y, z$.

\section{Experiment}

The formula (10) of spatial 3d picoscope was used to study images of single-layer and two-layer graphene taken on a transmission electron microscope with electron acceleration up to $80 \mathrm{kV}$. The image was obtained by Joerg R. Jinschek and others from FEI [7], Figure 5.

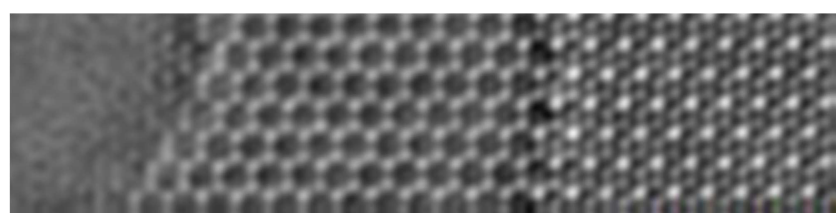

Figure 5. TEM image of single-layer and double-layer graphene [7].
It was found that the image in figure 6 cannot be obtained due to electron scattering, according to the authors [8]. The fact that scattering is a collective property of a large number of atoms, as shown in Figure 1(a). A separate electron cloud does not scatter the outer electrons.

Further conversion to direct electron density visualization was done using a picoscope [8], patent [6]. In view of the above theory, we obtained a spatial $3 \mathrm{~d}$ image of single-layer and two-layer graphene, shown in Figure 6.

From figure 6 it is clearly visible flat single-layer graphene, on the left. The carbon atoms are located in the same $x, y$ plane as hexagons of green color with the crystal lattice presented in [9].

On the right is the two-layer graphene, each third atom of which has a yellow color, which, according to formula (10), shows the exit of these atoms from the plane $x, y$ towards the observer by the height $\Delta z$.

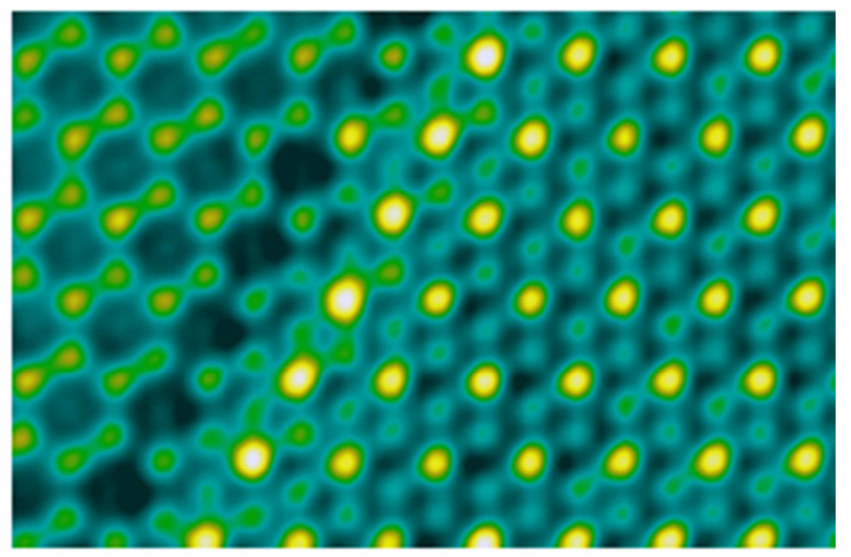

Figure 6. Direct spatial $3 d$ snapshot of single-layer graphene (left) and two-layer graphene (right), carbon atoms in the plane are green, close to an observer carbon atoms are yellow, and carbon atoms are distant from the observer are blue.

The image thus obtained clearly shows that the two-layer graphene react chemically, the $\pi$ - electron clouds join together and form the $s p^{3}$ bonds characteristic of the diamond. The carbon atoms in the lower layer are shifted to the bottom, and the atoms bound by $s p^{3}$ bonds rise above the plane to the height of $\Delta z$ and turn yellow.

\section{Interpretaition}

As a result, it was possible to trace a chemical reaction with a change in the chemical bonds, geometry molecules, and distances between atoms.

A graphical analysis of direct $3 \mathrm{~d}$ images of graphite and diamond is shown in figures 7 and 8 .

Figure 7 shows the intersection in the two-layer hexagonal diamond system (right), the carbon atoms in the plane are green, close to the observer the carbon atoms are yellow, and the atoms distant from the observer the carbon is blue. In the scheme: 1 - above the layer; 2 - top layer; 3 - the bottom layer; 4 - under the layer. It is the two-layer hexagonal diamond system that was previously unknown, which is proposed to 
designate gubanite.

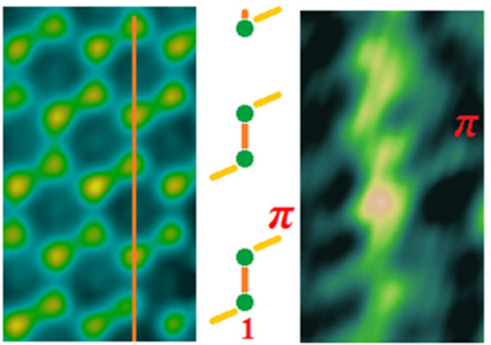

Figure 7. The scheme of intersection in the graphene layer, all the carbon atoms lie in the same plane and are green. The scheme shows the intersection alon red line 1: green indicated carbon atoms, red shows $\boldsymbol{s p}^{2}$ bonds of graphite, orange shows $\boldsymbol{\pi}$-electron clouds. On the right is picoscopic image of the intersection of the graphite layer [12,13].

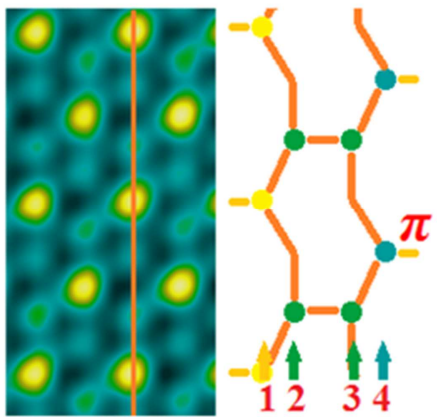

Figure 8. Direct spatial $3 d$ snapshot of the gubanite, the carbon atoms lying in the plane are green, the carbon atoms close to the observer are yellow, and the carbon atoms distant from the observer are blue. In the scheme: 1-above the layer; 2-top layer; 3- the bottom layer; 4- under the layer.

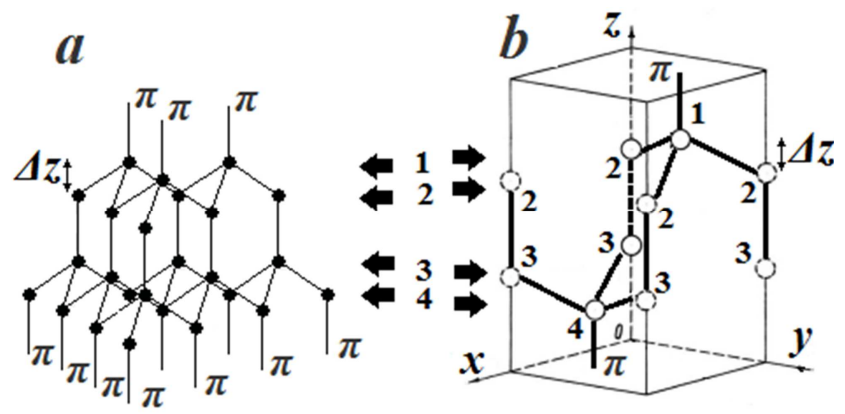

Figure 9. Model of crystal lattice of two-layer diamond (gubanite) (a). Structure of the gubanite elementary cell $\gamma-C(b)$, which coincides with the diamond elementary cell. Numbers indicate the layers of atoms: 2-top layer; 1- carbon atoms lying above the layer; 3- the bottom layer; 4-carbon atoms lying beneath the layer.

Figure 9 shows the crystal lattice of ordinary diamond (a) and two-layer diamond (gubanite) (b). The model shows carbon atoms located above the plane at the height $\Delta z$ found by the formula of the spatial $3 \mathrm{~d}$ pyroscope (2). Numbers indicate the layers of atoms: 2 - top layer; 1 - carbon atoms lying above the layer; 3 - the bottom layer; 4 - carbon atoms lying beneath the layer. The carbon atoms of the first and fourth layers of gubanite have $\pi$ - electron clouds that are not present in the crystal lattice of the diamond.

Figure 9 (c) shows the structure of the elementary cell of the gubanite crystal $\gamma-C$, which coincides with the elementary cell of the diamond.

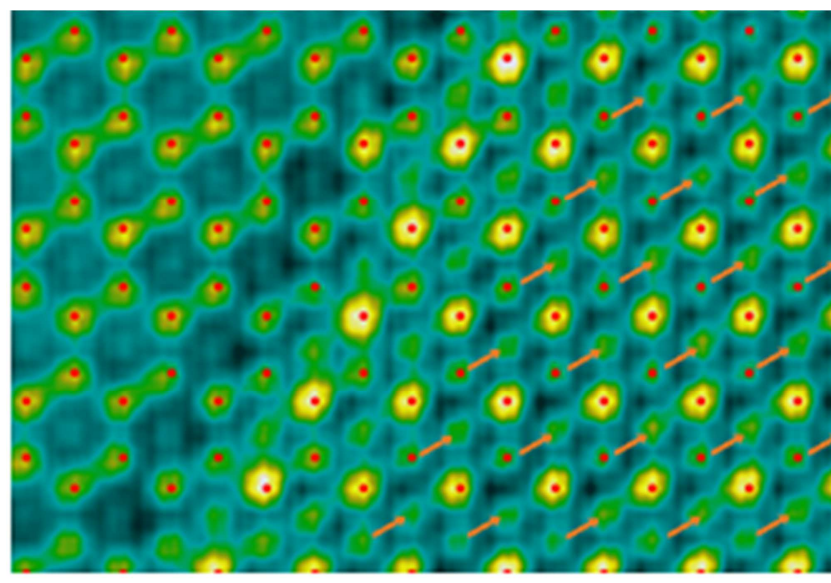

Figure 10. Direct spatial 3d snapshot of single-layer graphene (left) and two-layer graphene (right), arrows show the displacement of atoms in the formation of gubanite.

The transition from the flat crystalline lattice of graphene to the corrugated hexagonal lattice of diamond causes the appearance of $s p^{3}$ bonds and inverses the crystalline lattice of the lower gubanite, which we see in Figure 10. The shift of atoms is indicated by arrows.

It is important to note here that the diamond exists in another crystalline modification known as Lonsdaleite. Lonsdaleite was first identified in 1967 from the Canyon Diablo meteorite, where it occurs as microscopic crystals associated with diamond $[10,11]$. Structure diagrams of diamond and so-called lonsdaleite show their difference. Both consist of tetrahedrally coordinated carbon atoms that form layers. For ordinary diamond, the layers are oriented in the same direction but with so-called lonsdaleite, they are alternately oriented. From Figure 6 it is clearly visible that the layers are oriented in one direction, so it is diamond but not lonsdaleite.

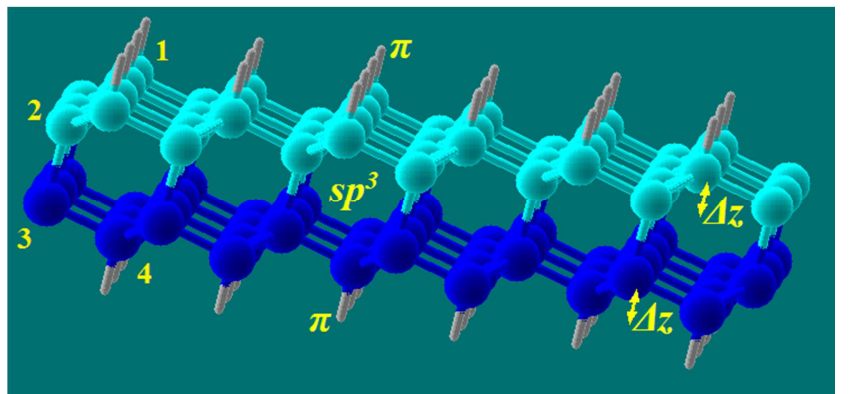

Figure 11. Spatial 3d model of the two-layer diamond-gubanite.

As can be seen from the spatial $3 \mathrm{~d}$ snapshot, Figure 10, as the graphene layers converge, the upper graphene $\pi$ - electron clouds connect to the lower graphene $\pi$ - electron clouds to form a two-layer diamond gubanite, the spatial $3 \mathrm{~d}$ model of which is shown in Figure 11.

Figure 11 shows a spatial $3 \mathrm{~d}$ model of a crystal lattice of a two-layer diamond (gubanite). The number 2 indicates the top layer and the number 3 indicates the bottom layer. The number 1 shows the atoms pushed over the top layer at the height $\Delta z$. The number 4 shows the atoms pushed under the lower layer by the height $\Delta z$. The carbon atoms located in layer 1 have an 
upward $\pi$ - electron clouds, and the carbon atoms located in layer 4 have a downward $\pi$ - electron clouds.

\section{Conclusions}

From the theory of the electron beam shifting effect the formula of spatial $3 \mathrm{~d}$ snapshot of electron clouds of atoms is obtained. This formula was used to analyze the spatial arrangement of atoms in graphene and gubanite layers. The case of the transformation of two layers of graphene into a diamond crystal lattice - gubanite is presented.

In his lectures on quantum mechanics, R. F. Feynman [14] said:" We know how large objects will act, but things on a small scale just do not act that way. So we have to learn about them in a sort of abstract or imaginative fashion and not by connection with our direct experience." With the opening of the picoscope, the time of "abstract or imaginative fashion" passed - from now on we have the opportunity to actually see the small-scale objects and we can study them by using our direct experience. This is a fundamental step towards understanding the logic of behavior of the molecular world around us. The era when the teachers talk about molecules, that they have never seen them, has ended. The era of unity with nature, consisting entirely of molecules, has begun. The appearance of visual chemistry is our way.

\section{References}

[1] C. Lenardi, P. Piseri, V. Briois, C. E. Bottani, A. LiBassi, P. Milani, J. Appl. Phys. 85 (1999) 7159.

[2] S. diStasio, A. Braun, Energy Fuels 20 (2006) 187.

[3] Adriyan Milevetal. Influence of bond defects on coiling of graphite. 2006 Sci. Technol. Adv. Mater. 7, 834.

[4] O. P. Kucherov \& A. D. Rud (2018) Direct visualization of individual molecules in molecular crystals by electron cloud densitometry, Molecular Crystals and Liquid Crystals, 674: 1, 40-47, https://DOI:10.1080/15421406.2019.1578510.
[5] O. P. Kucherov, \& S. E. Lavrovsky, Direct visualization of molecular structure by the electron beam shifting effect. Information Technology and Special Security, (2016). (http://science-ua.com/gallery/maketn2.pdf).

[6] O. P. Kucherov, \& S. E. Lavrovsky. The method for obtaining an image with a sub-diffraction resolution and an opto-electronic system for its implementation, Patent of Ukrainen 115602 dated February 27, 2018.

[7] Jinschek, J. R.; Yucelen, E.; Calderon, H. A.; Freitag, B. Quantitative atomic 3-Dimagingofsingle/ double sheet graphene structure. Carbon 2011, 49, 556-562.

[8] O. P. Kucherov, \& S. E. Lavrovsky, Picoscope, As an Instrument for Molecules Atomic Structure Study. Information Technology and Special Security, (2016). (http://science-ua.com/gallery/maketn2.pdf).

[9] V. O. Gubanov, A. P. Naumenko, M. M. Bilyi, I. S. Dotsenko, O. M. Navozenko, M. M. Sabov, L. A. Bulavin. Energy spectracorrelation of vibrational and electronic excitations and their dispersion in graphite and graphene// Ukr. J. Phys. 2018.

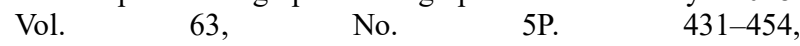
https://doi.org/10.15407/ujpe63.5.431.

[10] Frondel, C.; U. B. Marvin (1967). "Lonsdaleite, a hexagonal polymorph of diamond". Nature. 214 (5088): 587-589. Bibcode: 1967 Natur. 214.587F. http://doi:10.1038/214587a0.

[11] Frondel, C.; U. B. Marvin (1967). "Lonsdaleite, a hexagonal polymorph of diamond". American Mineralogist. 52.

[12] A. D. Rud, N. E. Kornienko, I. M. Kiryan, A. N. Kirichenko, \& O. P. Kucherov. (2018). Local heteroallotropic structures of carbon. Materials Today: Proceedings, 5, 26089-26095.

[13] A. D. Rud, I. M. Kiryan, \& A. M. Lakhnik, Topological characteristics of local atomic arrangements at crystalline-amorphous structural transition in graphite. Materials Science. Mesoscale and Nanoscale Physics (2014). (http://arxiv.org/abs/1412.1982.

[14] Feynman Lectures on Physics, Quantum Mechanics. PaloAlto, London, $1961 . \quad 325 \mathrm{p}$ (https://www.academia.edu/28997196/The_Feynman_Lecture s_on_Physics_-_VOL3). 